\title{
Design and evaluation of 3D cursors and motion parallax for the exploration of desktop virtual environments
}

\author{
David Antonio Gómez Jáuregui* $\quad$ Ferran Argelaguet $^{\dagger} \quad$ Anatole Lecuyer $^{\ddagger}$
}

INRIA Rennes

\begin{abstract}
The selection and manipulation of 3D content in desktop virtual environments is commonly achieved with 2D mouse cursor-based interaction. However, by interacting with image-based techniques we introduce a conflict between the $2 \mathrm{D}$ space in which the $2 \mathrm{D}$ cursor lays and the 3D content. For example, the 2D mouse cursor does not provide any information about the depth of the selected objects. In this situation, the user has to rely on the depth cues provided by the virtual environment, such as perspective deformation, shading and shadows.

In this paper, we explore new metaphors to improve the depth perception when interacting with 3D content. Our approach focus on the usage of $3 \mathrm{D}$ cursors controlled with $2 \mathrm{D}$ input devices (the Hand Avatar and the Torch) and a pseudo-motion parallax effect. The additional depth cues provided by the visual feedback of the $3 \mathrm{D}$ cursors and the motion parallax are expected to increase the users' depth perception of the environment. The evaluation of proposed techniques showed that users' depth perception was significantly increased. Users were able to better judge the depth ordering of virtual environment. Although 3D cursors showed a decrease of selection performance, it is compensated by the increased depth perception.
\end{abstract}

Index Terms: H.5.2 [Information interfaces and presentation]: User Interfaces-Evaluation/methodology; I.3.6 [Computer Graphics]: Methodology and Techniques-Interaction techniques;

\section{INTRODUCTION}

The interaction with $3 \mathrm{D}$ virtual worlds is becoming more and more common. The arrival of HTML 5.0 and WebGL is unleashing the exploration of the 3D worlds directly from a standard web browser. However, interaction in desktop virtual environments (DVE) is still achieved through image-based techniques. Image-based techniques do not reinforce the depth perception of the user as the visual feedback is displayed in a plane perpendicular to the user's viewing direction. Unfortunately, most 3D commercial applications for computer aided design and video-games still employ this approach.

User's depth perception plays an important role in building spatial relationships inside a virtual environment. Psychologists have systematically explored how people perceive their surroundings and how they retrieve depth information. Their findings have been widely employed to increase the depth perception of 3D computer generated imagery. Depth cues such as perspective deformation, occlusion and shading are supported by hardware for all rendering APIs (e.g. OpenGL). In addition, more advanced lighting and optical effects, such as physically based shading, shadows and depthof-field effects can also be added. However, this information is not

*e-mail: david.gomez_jauregui@inria.fr

†e-mail: fernando.argelaguet_sanz@inria.fr

†e-mail:anatole.lecuyer@inria.fr reinforced by a $2 \mathrm{D}$ cursor which is always displayed at the imageplane, it might even break the depth illusion. Several studies have proven that $3 \mathrm{D}$ object selection metaphors, such as raycasting and the virtual hand, allow users to perceive and interact with 3D content in a more natural and consistent way. However, such metaphors require $3 \mathrm{D}$ input devices, which generally lack the precision and stability of a mouse [6].

In this work, we describe new interaction techniques focused on the increase of the user's depth perception when interacting with desktop virtual environments. These techniques will take into account the underlying 3D content of the DVE in order to provide a more "ecological" interaction. First, we propose two new 3D cursor metaphors controlled by two-dimensional input devices: the Hand Avatar and the Torch. Both, display 3D visual feedback according to the 3D point determined by the mouse cursor, the virtual camera and the virtual environment. Furthermore, we explore an orthogonal approach to increase depth perception, the pseudo-motion parallax. Motion parallax is the phenomenon in which a change of the user's position in a stationary environment results in the perception of apparent motion of objects in the environment [23]. Both approaches are expected to have additive effects on the increase of the users' depth perception. The theoretical analysis is accompanied with a user evaluation exploring how the proposed techniques may affect object selection tasks in DVEs. The user study explored whether selection performance and depth perception is improved or degraded in comparison with traditional 2D selection techniques.

The rest of the paper is organized as follows. Section 2 reviews related work on 3D selection techniques, depth perception and the role of visual feedback. Section 3 describes the proposed metaphors for depth perception enhancement. We present the user evaluation results in Section 4, and the paper ends with the conclusions in Section 5.

\section{Related Work}

Web technologies are evolving rapidly from 2D content navigation (HTML, XML) to 3D interactive content (WebGL, X3D, XML3D) [24]. However, 2D object selection metaphors do not exploit the third dimension, since objects are selected only considering its projection. In the following, we review existing alternatives for $3 \mathrm{D} / 2 \mathrm{D}$ object selection, the role of the visual feedback provided during a selection task and how depth perception can be enhanced in computer generated imagery.

\subsection{Object Pointing}

Pointing to a 3D object in a desktop virtual environment is commonly accomplished by placing the mouse cursor over the desired target and pressing a mouse button. Improvements on mousebased selections have been mainly focused on improving user performance, by exploiting Fitts' law [9] principles. Such improvements are based on increasing the size of the cursor, decreasing the distance between targets or adapting the CD ratio [4]. Twodimensional pointing techniques mainly focus on 2D Graphical User Interfaces, with few exceptions such as semantic pointing for 3D object selection [8]. However, current 2D object selection techniques for $3 \mathrm{D}$ content do not take into account the underlying $3 \mathrm{D}$ 
content. Existing techniques do not provide any information regarding the 3D position of the object being selected.

In contrast, 3D object selection techniques for immersive virtual environments (IVEs) take into account the third dimension, but they require free-hand interaction like Raycasting and Virtual Hand metaphors [5]. They had specific requirements as the overall difficulty of the task increases due to the additional degrees of freedom and the absence of physical constraints [4, 12], and thus an increased user effort. Similar to 2D pointing facilitation techniques, techniques to improve $3 \mathrm{D}$ object selection rely on increasing the size of the cursor at the expense of disambiguation mechanisms [18], CD ratio adjustments [10] or statistical approaches based on users' actions [7].

However, although 3D object selection techniques are aware about the underlying 3D content, they usage in a desktop environment is limited due to the required input devices. In contrast, 2D cursor based techniques are discouraged when using stereoscopic displays [3]. The 2D cursor would conflict with the stereoscopic image, resulting in depth cue conflicts and reduced pointing performance.

\subsection{Depth Perception}

The ability to retrieve depth information from a virtual environment allows the observer to better understand the relationship between objects. In the absence of depth cues, ambiguity may arise and different users may perceive differently the same virtual environment [29].

Depth cues, according to psychologist, are classified into two groups, primary and pictorical cues. While primary cues relate to physiological aspects such as the binocular disparity and eye accommodation, and can only be achieved in true 3D vision, pictorical cues (e.g. perspective deformation, shadows, occlusion) can be simulated to create the illusion of depth. Computer generated images which already include perspective deformation and occlusion cues can be enhanced with physically based rendering such as shadows and complex lighting effects.

As we are targeting 2D input and output devices, primary depth cues cannot be employed to boost the observer's depth perception, but pictorical depth cues can. Wanger et al. in [29] explored how the combination of pictorical depth cues can enhance depth perception. Their results showed that shadows and perspective were the strongest cues for depth perception. Shadows can be easily simulated in modern graphics hardware, and in addition to increase depth perception [14], it can also improve the perceived distance among two objects [13] and shape recognition [28].

Motion can also enhance the depth perception, either by motion parallax [23] (changing the observer's position) or through the kinetic depth effect [2] (e.g. the continuous rotation of an object along its vertical axis). However, as we want to keep the virtual environment unaltered, we consider only motion parallax.

Motion parallax produces consistent and unambiguous impression of depth [30] even if no other cue is present [23]. Motion parallax is obtained through tracking the observer's viewpoint, and adapting the virtual camera to match the observer's physical position. Another alternative, is to induce motion parallax by simulating the effect of the user moving. Kitazaki et al. in [17] were able to induce motion parallax effects by changing the virtual camera position in the horizontal axis (1 meter distance at $0.4 \mathrm{~Hz}$ ).

\subsection{Visual cursors}

Cursors provide the visual feedback to allow the user to drive the input device to select the desired object. The most common 2D cursor is an arrow-shaped cursor with an upper-left orientation. Its shape can be designed to change automatically according to users' actions, thus providing context-sensitive information. For example, the cursor might turn into a pointing hand if it is over an hyper-link or into a typewriter cursor if it is inside an editable text area. However, although there are several studies on 2D cursor shapes [20,21], they are only focused on 2D content.

In contrast, 3D object selection techniques for IVEs provide visual feedback adapted to 3D content (except for image-based techniques). The visual representation of 3D cursors mainly relate to geometrical shapes such as rays, cubes or visual representations of the user (e.g. hand avatar). However, we have to be careful when determining the shape of the 3D cursor, Kadri et al. in [16] showed that for 3D manipulation tasks, the visual appearance influenced users' actions. The shape of the $3 \mathrm{D}$ cursor can determine how the user manipulate virtual objects.

The following questions arises, can we efficiently control a 3D cursor only through two degrees of freedom? A 3D cursor controlled through two degrees of freedom will provide a more ecological way to interact with $3 \mathrm{D}$ content and might introduce additional depth cues.

\section{Novel 3D CURSORS AND PARALLAX EFFECT FOR EXPLORING 3D CONTENT USING A MOUSE}

In this section, we explore several alternatives to improve the users' depth perception of 3D environments. We first detail our proposed 3D cursor methapors and then detail the pseudo-motion parallax approach. We believe that our techniques will increase the depth perception of 3D content when interacting with a $2 \mathrm{D}$ input device.

\subsection{D Cursors}

The classification of visual feedback approaches for 2D mousebased selection techniques has two main groups: image-based and projection-based techniques. Visual feedback approaches for image-based techniques consider only the image obtained by the projection of the 3D scene into the projection plane. The more common example is standard 2D mouse-based pointing; the image of the mouse pointer is overlaid on top of the $2 \mathrm{D}$ projection of $3 \mathrm{D}$ scene. Although additional picking strategies can be employed, such as semantic pointing [8], these approaches do not take advantage of the three dimensions of the environment, and do not provide any reliable information about depth information.

In contrast, projection-based approaches display the visual feedback directly on the 3D scene, thus taking into account the 3D information provided by the environment, and not only its projection. Several projection-based approaches has been developed for IVEs [3]. However, its usage with 2D input devices (e.g. the mouse) is limited. Stenicke et al. [25] proposed the 3D Depth Cursor. In their approach the mouse cursor was always displayed on top of the objects' surface. However they did not detail how the cursor was projected into the $3 \mathrm{D}$ content and was not formally evaluated.

We propose two novel approaches: the Torch and the Hand Avatar, which explore the combination of image and projectionbased.

\subsubsection{Torch}

In the Torch metaphor, we were interested to explore whether the combination of image-based and projection-based approaches reinforce the depth perception of the user without sacrificing the efficiency of 2D cursors in terms of selection time and accuracy. We chose this metaphor since it provides a more natural way to explore $3 \mathrm{D}$ content. Figure 1 depicts the different components of the visual feedback provided.

Two projection-based components are employed: the light cone and the specular highlight. The light cone generates a halo of light that gradually becomes wider with increasing depth distance. In addition, a linear decay of the brightness of the light cone was used to decrease its brightness as the depth increases. A specular highlight is simulated by drawing a 2D disk on the surface of the selected object, the disk is drawn semi-transparent in order to not occlude the 


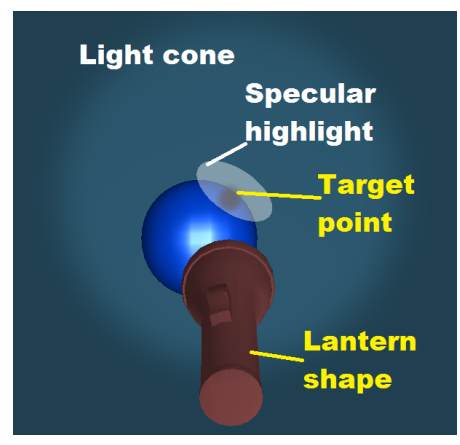

Figure 1: Visual feedback provided by the Torch metaphor. The blue sphere is the selected object. The projection-based components (white) provide additional visual feedback about the shape and depth of the selected 3D object. The image-based techniques (yellow) reinforce the metaphor (lantern shape) and improve the accuracy (target point).

selected object. Finally, it is also oriented according to the normal of the surface selected point, thus providing a visual feedback about the shape of the object.

As image-based components, we used a lantern-shaped cursor and a target point. While the purpose of the lantern-shaped cursor is to reinforce the torch metaphor, the target point was introduced to avoid ambiguities. A general problem with the light cone is that multiple objects can be intersected or fall within the cone area [26]. The target point is drawn according to the coordinates of the mouse pointer, which also matches to the center of the specular highlight.

The depth cues introduced by the torch are those provided by the projection-based components. The light cone introduces two depth cues, the size of the halo and its brightness. The specular highlight introduces a perspective depth cue (its projection gets smaller as the distance increases, see Figure 2 top).

Implementation details. The update of the specular highlight require the computation of the projected point and its normal every frame. In order to reduce the possible overhead, the depth and the normal can be fetched from an off-screen buffer containing a depth and a normal map of the scene. These buffers can be easily computed during the rendering process.

\subsubsection{Hand Avatar}

In this approach, we only employed projection-based visual feedback. The virtual hand metaphor, has been widely employed for the selection of 3D objects in IVEs. A 3D cursor (e.g. hand avatar) is governed by three degrees of freedom, which allows the user to "touch" objects. However, its usage was not intended to use in combination with a input device with only two degrees of freedom. Although some studies about 3D hand metaphors have been done using 3D input devices (e.g. PHANTOM device) [15], [27], as far as we know, no study has been done on using such a hand avatar metaphor with 2D input devices (e.g. mouse).

For our approach, the visual feedback is provided by displaying a 3D hand avatar on the surface point determined by the projection of the mouse cursor. The surface point is computed by casting a ray with its origin determined by the position of the mouse cursor and with an orientation parallel to the viewing direction. As the environment is displayed with perspective projection and we keep the size of the avatar unchanged, the relative size of the hand decreases as the depth increases. Perspective is a strong depth cue, which will allow the user to infer depth information according to the displayed size of hand avatar (see Figure 2 bottom). In this approach, a limitation of the visual feedback may exist with respect to huge depth ranges, for example, if the depth distance of a selected object be-
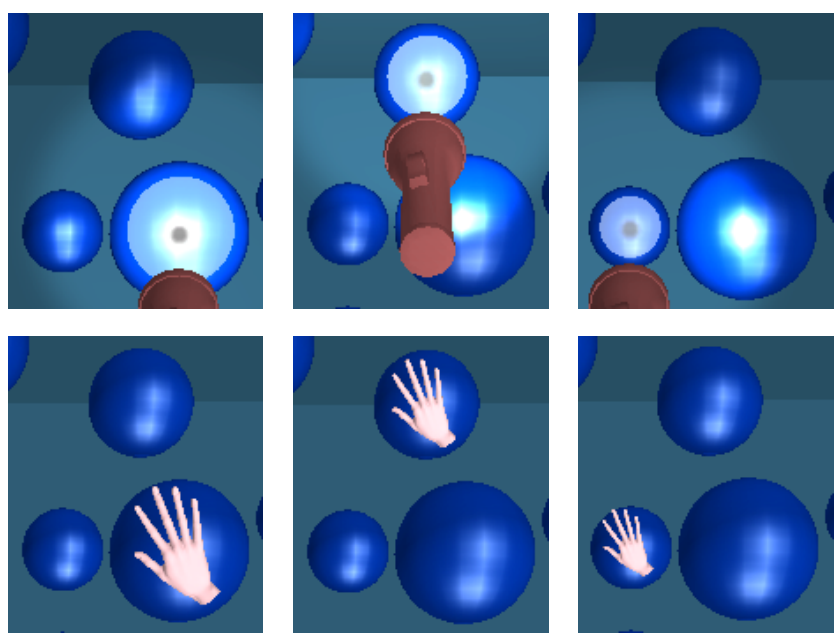

Figure 2: The relative size of the specular highlight (top) and the hand avatar (bottom) allows the user to judge relative depth disparities between $3 \mathrm{D}$ objects. From left to right, the distance towards the observer increases.

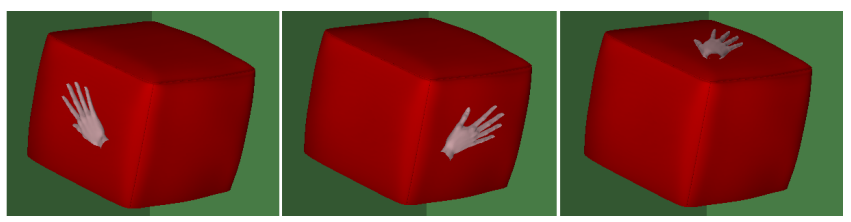

Figure 3: The hand avatar is positioned and rotated according to the position and the normal of the surface, thus giving the pseudo-illusion of touch.

comes infinitely large, the hand size will become infinitely small. In these cases, the hand will be projected in a smaller depth distance with respect to the depth of the selected object. In this way, the virtual hand is defined with a maximum depth range. The color of the hand avatar can be changed in order to warn the user.

In order to minimize the penetration of the hand avatar with the virtual environment, we also rotated the hand avatar by following the surface normal of the object. The palm of the hand will always face the selected object in order to provide a pseudo-illusion of touch with standard mouse interaction (see Figure 3). The change of orientation of the hand avatar provides additional information about the shape of $3 \mathrm{D}$ objects.

Although the hand metaphor may provide a some complex visual feedback, we believe that is necessary to combine both visual cues (rotation and perspective projection) in order to provide an intuitive and natural interaction with the 3D objects since the visual feedback resembles the real life action of touching an object.

\subsection{Pseudo-Motion Parallax}

The Hand Avatar and the Torch, allow to locally inspect each object of the virtual environment in order to infer depth information. However, users need to "touch" all objects if they want to gather depth information of all the environment.

An orthogonal approach to increase depth perception is the use of motion parallax. By adding a motion parallax effect, which has been shown to effectively increase depth perception [30,17], we aim to provide the user with a global approach to infer depth information. The pseudo-motion parallax effect will simulate movements of the observer constrained in a plane perpendicular to the viewing direction. The effect is obtained by translating the virtual camera in the viewing plane (see Figure 6). Consider that the 
pointer is in the center of the viewpoint, cursor movements to the left or the right, will result in camera translations to the same direction, the same applies to upwards and downwards movements. The null compliance is maintained, when the cursor returns to the center of the viewport the accumulated translation is zero.

The camera motion will result in displacements for all the object's projection in the environment $\left(\Delta P_{\text {screen }}\right)$, although not uniformly. The displacement of an object is proportional to the mouse cursor movement $\left(\Delta P_{\text {mouse }}\right)$ and inversely proportional to the depth value of the object $(d)$ (see Equation 1). It is also dependent of the field of view of the virtual camera $\alpha$.

$$
\Delta P_{\text {screen }}=\frac{\Delta P_{\text {mouse }}}{2 d \tan (\alpha / 2)}
$$

The theoretical analysis on the possible impact on selection performance follows. On the one hand, as the cursor approaches the target, the object's projection will slightly approach the cursor (see Figure 4 left). On the other hand, once the cursor is inside the target, the camera movement will decrease the size of the target in motor space (see Figure 4 right). Following Fitts' law notation [9],

$$
M T=a+b \log _{2}\left(\frac{D}{W}+1\right)
$$

the pseudo-motion parallax effect will equally decrease the amount of distance the cursor has to cover $(D)$ and the size of the target $(W)$. In some sense, it changes the CD ratio, but keeps the index of difficulty unchanged.

However, as depicted in Figure 5, the CD ratio decreases with respect to the depth position of the target (the field of view depends on the application setup). While a target placed at the infinity will keep a 1:1 CD ratio, the closer the target the lower the $\mathrm{CD}$ ratio will be. If the user does not compensate for the movement, the movement of the object's projection might hinder the pointing process. The user might over estimate the movement required to reach the target, requiring additional corrective movements. For example, if the camera movement is too high, it will be harder for the user to estimate the mouse movement required to aim a target. On the other hand, if the camera movement is too low, the depth information provided will be reduced. The mapping can be adjusted considering the depth range of the 3D scene.

Our approach has some similarities with the effects obtained with the parallax scrolling techniques, which has been employed in $2 \mathrm{D}$ games and web pages to create a depth illusions. However, as far as we know, these approaches do not simulate real virtual camera movements and there is not any study in the literature exploring its usage for $2 \mathrm{D}$ selection tasks.
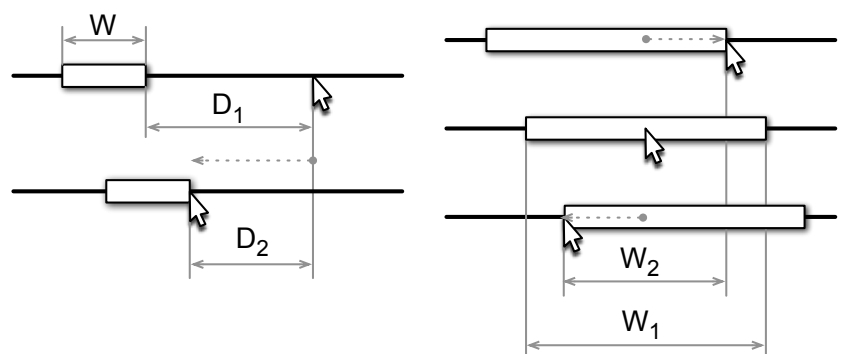

Figure 4: (Left) The pseudo-motion parallax approach reduces the distance covered by the mouse cursor when aiming to a target. When the cursor approaches the target, the target also approaches the cursor $\left(D_{2}<D_{1}\right)$. (Right) The pseudo-motion parallax approach also reduces the effective size of the target. When the cursor is inside the target, the movement of the target goes in the opposite direction of the cursor movement $\left(W_{2}<W_{1}\right)$.

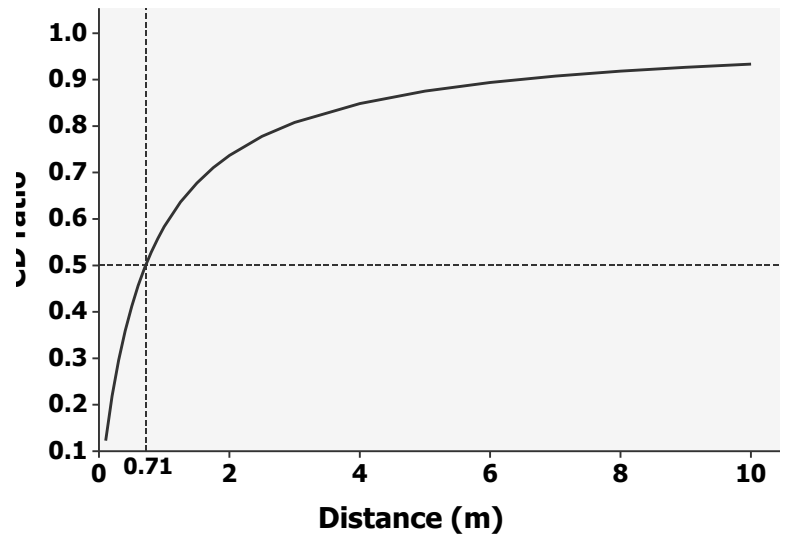

Figure 5: Change of the CD-ratio as the distance to the target increases when the fov is fixed at 70 degrees. Objects at $0.71 \mathrm{~m}$ have a CD ratio of 1:2.

\section{User Evaluation}

We conducted a user evaluation in order to explore the viability of proposed techniques, exploring their potential benefits and drawbacks. The main difference from 2D cursor-based techniques is that the proposed visual feedback is 3D content friendly, as it adapts to the 3D content. While the Torch alters the visualization, the Hand Avatar allows the user to "touch" objects. Moreover, the pseudomotion parallax effect can be combined with any visual feedback approach, simulating that the user is moving his head to explore the virtual environment. During the evaluations users were instructed to perform two different tasks, one to evaluate the selection performance and a second one to explore the additional depth information gathered with the proposed techniques.

On the one hand, the addition of visual feedback and the motion parallax effect may increase the user's depth perception. On the other hand, the increase may be non-significant, as pictorical depth cues such as occlusion and perspective deformation may provide comparable results, and selection performance might decrease. The cost of actively exploring the environment in order to obtain additional depth cues may introduce excessive overhead, discouraging their usage. In addition, camera movements might produce distracting effects, reducing user performance when performing selection operations. If users are not able to compensate for camera movements they would have to perform additional corrective movements. Figure 6 shows an example of the maximum camera displacement used during the evaluation. Furthermore, increasing the amount of visual feedback does not always improve user performance [22] and might even reduce selection performance [11].

Although other depth perception studies employ shadows to introduce additional depth cues $[29,14]$, we decided not to use them. Depth perception studies tend to use shadows casted perpendicularly to the ground, which may not be the case for general 3D environments. Also, the study presented by Hubona et al. in [14] showed that the effect of shadows tends to decrease when more than one light source casts shadows or the floor is not regular, which may also be the case in a general 3D environment.

\subsection{Procedure}

Users were presented with several synthetic desktop virtual environments containing spheres placed at different depth values (see Figure 6). The potential targets were contained within a virtual room to provide the user with a fame of reference. The scene is rendered using a perspective projection and objects are shaded using Phong shading. Users had to perform two different tasks. 

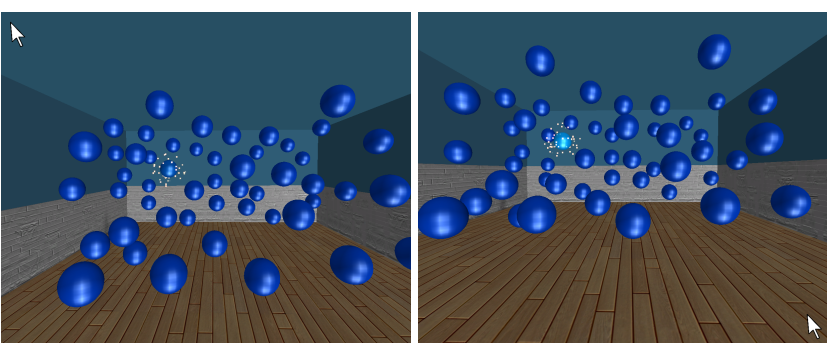

Figure 6: Test environment used during the first task of the user evaluation. Both images show the maximum camera displacement perceived by the user when the pseudo-motion parallax effect was enabled. Left, the mouse is at top left corner. Right, the mouse is at the bottom right corner.

The first task was meant to evaluate pure point-and-click performance. Proposed alternatives in comparison to $2 \mathrm{D}$ cursors, might negatively impact user performance during fast aimed movements. The task consisted in selecting a sequence of objects where the next object to be selected was clearly highlighted. The test environment consisted in a cubical room with fifty spheres. The position of the spheres were computed randomly, but as the user's viewpoint was constraint, we adjust the horizontal and vertical position of the spheres to minimize occlusion among them (see Figure 6). If all the spheres are visible from the user's viewpoint, we avoid bias due to object discovery. We pre-computed a random sequence of sixty targets; the same sequence was used for each trial. The first ten targets were considered as training.

In contrast, the second task was meant to evaluate the users' depth perception of the environment. In the best case scenario we expected the $3 \mathrm{D}$ cursors and the motion parallax to have additive effects. Users were informed to sort all the objects inside the environments according to their depth value. The user had to click all objects, starting from the closest one and ending with the furthest one. Users were not able to undo any selection. Six different test environments were used, each containing ten spheres. Although the relative depth-size cue was not provided, the size of the spheres was not uniform, it held for most of the spheres. For each condition, the first environment was a training exercise, in which users were instructed to explore how they could take advantage of each technique. The training exercise was always done with the same environment and the remaining five were randomly presented to users.

\subsection{Design and Hypotheses}

For the first task, a repeated-measures within-subjects design was used. The independent variables were the interaction technique used: Hand Avatar (HA), Torch (TO) and the 2D Cursor (2D), and the camera motion: static camera (SC) and motion parallax (MP). The order of the factors were counter-balanced using a Latin Square design to avoid ordering effects. The dependent variables were the selection time and the deviation in pixels from the selection point to the center of the target. Users were informed that the selection speed was more important than their accuracy, but they were also advised to keep the number of errors within a threshold. They were not explicitly asked to aim to the center of the spheres. Our hypotheses regarding the first task were:

H1.1 : Lower mean of selection time in the 2D Cursor condition as compared to both of the remaining conditions.

H1.2 : Higher mean of selection time in the motion parallax condition.
H1.3 : Lower mean of pixel deviation in the 2D Cursor condition as compared to both of the remaining conditions.

H1.4 : Higher mean of pixel deviation in the motion parallax condition.

For the second task, a repeated-measures within-subjects design was also employed. The independent variables were also the technique: HA, TO and 2D, and the camera motion: SC, MP. The order of the factors were also counter-balanced using a Latin Square design. The dependent variables were the time-to-complete the task and the ordering error. The ordering error $(S)$ was computed as follows,

$$
S=\sum_{i=0}^{10}\left|i-i_{\text {res }}\right|^{2}
$$

being $i$ the position of the $\mathrm{i}$-th object in the ordering, and $i_{\text {res }}$ the position of the i-th object in the user's ordering. For comparison purposes, a random sorting will obtain a mean of approximately 164. Users were informed that was preferred to obtain a good ordering rather than a fast task completion time. Our hypotheses for the second task were:

H2.1 : Lower mean of task completion time in the 2D Cursor condition as compared to both of the remaining conditions.

H2.2 : Lower mean of task completion time in the motion parallax condition.

H2.3 : Higher mean of ordering error in the 2D cursor condition as compared to both of the remaining conditions.

H2.4 : Lower mean of ordering error in the motion parallax condition.

Users performed first the point-and-click task followed by the ordering task. At the end of each task, a short questionnaire was given to users to obtain subjective ratings of the techniques. Users had to rank each question using a 5-likert scale. Each participant performed the experiment in one session lasting approximately 25 minutes.

\subsection{Apparatus and Participants}

The experiments were conducted in a 24 " inch monitor with a resolution of $1920 \times 1200$ pixels and using as input device a standard mouse. The prototype was implemented using Unity 3D [1] and the frame rate was stable at 60 FPS for all conditions. Twelve volunteer users (undergraduate and graduate students) from our lab, which did not have previous knowledge of the evaluated techniques participated in the study. Aged from 22 to 34, 10 male and 2 female. 5 participants play video games more than 3 times per week; 4 play video games at least 1 time per week and 3 participants do not usually play video games.

\subsection{Results}

\subsubsection{Selection Task}

Data obtained for each user was collapsed and entered into a twofactor (technique $\times$ camera) within-subjects ANOVA. For all posthoc comparison, Tukey comparisons are applied.

Regarding selection time (see Figure 7 Top), the analysis showed main effects for technique $(p<0.001 ; F=537.83)$. Post-hoc tests revealed that all techniques were significantly different (all $p<0.05$ ). Users performed faster with the $2 D$ Cursor followed by the Torch and the Hand Avatar. This confirms H1.1, stating that users performance would be higher with the standard mouse 

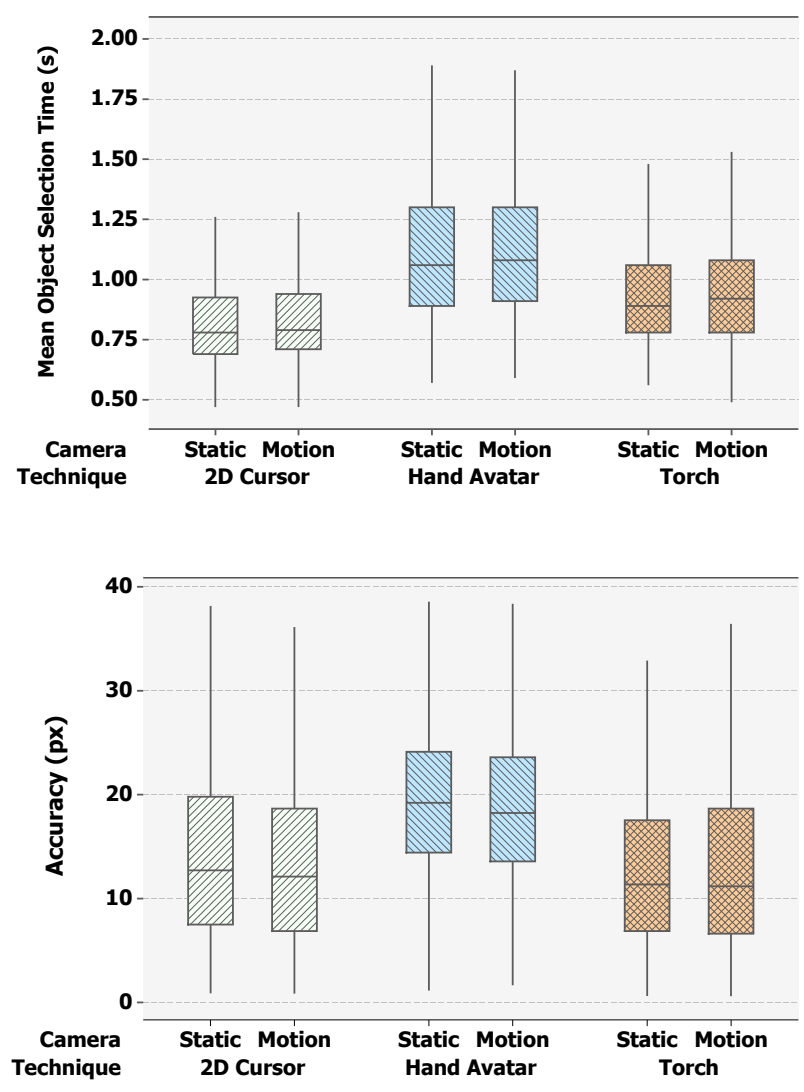

Figure 7: Boxplots of selection time (top) and selection accuracy (bottom) corresponding to the results of the first task. The effect of the camera factor for selection time and accuracy was found nonsignificant.

cursor. In contrast, no main effects were found for the camera factor $(p=0.126)$, thus rejecting H1.2. The pseudo-motion parallax effect did not increase selection time. Any interaction effect was found among factors $(p=0.382)$.

With respect to selection accuracy (see Figure 7 Bottom), there was a main effect for technique $(p<0.001 ; F=152.05)$. Posthoc comparisons showed that the Hand Avatar condition presented the lowest accuracy followed by the $2 D$ Cursor $(p<0.05)$, the Torch got the best accuracy $(p<0.05)$. With this results we partially reject $\mathrm{H} 1.3$, the $2 D$ Cursor still presented a better behavior than the Hand Avatar. On the other hand, the camera factor did not present any main effect ( $p=0.326)$, thus rejecting H1.4. The pseudo-motion parallax effect did not decrease user accuracy. The interaction among factors was also non-significant $(p=0.420)$.

\subsubsection{Ordering task}

Data obtained for each user was collapsed and entered into a twofactor (technique $\times$ camera) within-subjects ANOVA. For all posthoc comparison, Tukey comparisons are applied.

Regarding ordering time (see Figure 8 Top), the analysis showed a main effect for technique $(p<0.001 ; F=96.93)$. Post-hoc comparisons showed that all techniques presented significant differences (all $p<0.05$ ). Users performed faster with the $2 D$ Cursor, followed by the Torch and the Hand Avatar. This confirms H2.1, being the 2D Cursor faster than both 3D Cursors. In contrast, there was no main effect for camera $(p=0.697)$, thus rejecting $\mathrm{H} 2.2$. However, an interaction effect was found for technique and cam-
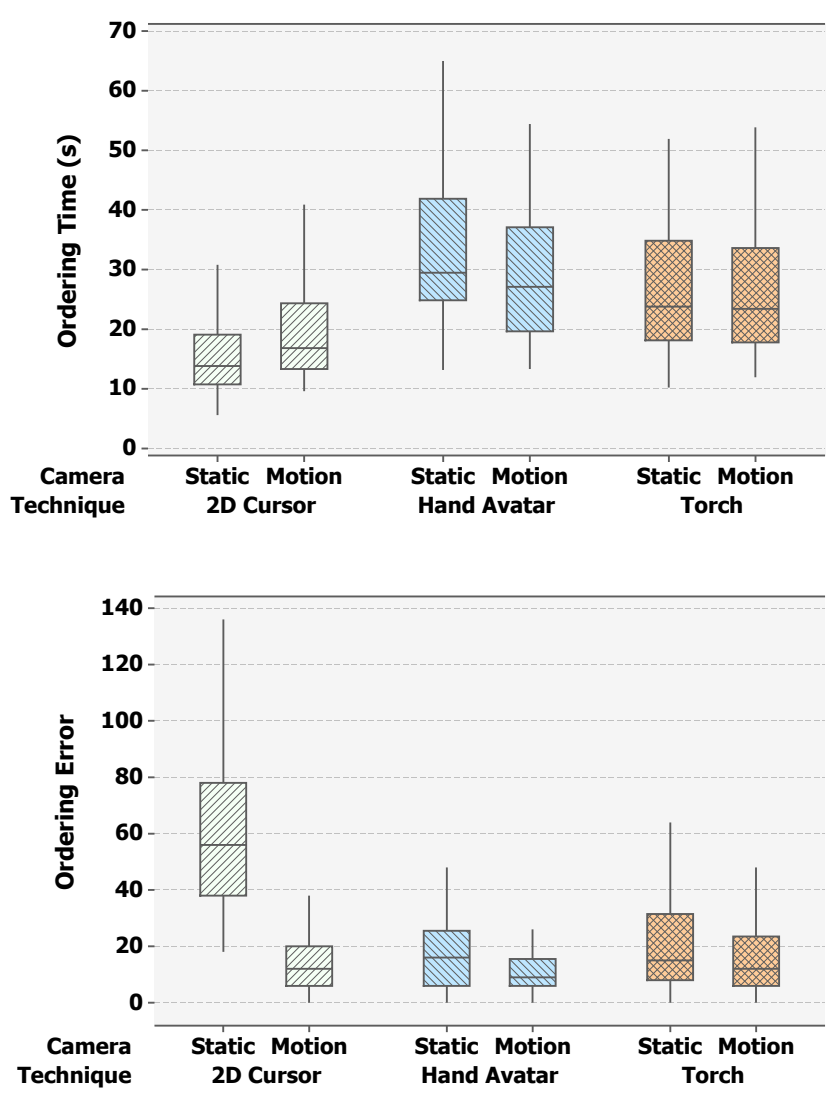

Figure 8: Boxplots of ordering time (top) and error (bottom) corresponding to the results of the second task. In overall, the addition of depth cues helped in the sorting of the scene, but they also increased the amount of time required to perform the task.

era $(p<0.001 ; F=10.27)$. Post-hoc analysis revealed that for the Hand Avatar condition the task completion time was significantly lower in combination with the Motion Parallax condition $(p<0.05)$. Users were able to sort objects faster with the pseudomotion parallax effect using the Hand Avatar, however no such effect was observed for the Torch.

With respect to the ordering error (see Figure 8 Bottom), there was also a main effect for technique $(p<0.001 ; F=23.98)$. Posthoc comparisons showed that there were significant differences between the $2 D$ Cursor condition and both of the 3D Cursor techniques (all $p<0.05$ ). This confirms H2.3. Also, there was a main effect for the camera $(p<0.001 ; F=75.40)$, when the pseudomotion parallax was enabled, users did less errors when sorting the objects, thus confirming H2.4. Finally, the ANOVA analysis also showed an interaction effect for technique and camera conditions $(p<0.001 ; F=31.57)$. The post-hoc analysis showed that the combination of the $2 D$ Cursor and the static camera had the highest mean of ordering error. Also, the combination of the Torch and the static camera had a higher mean of ordering error than the combination of the Hand Avatar and the motion parallax $(p<0.05)$.

\subsubsection{Questionnaires}

After the completion of each task, we gave users a questionnaire to know their preferences. At the end of the selection task, users were asked to sort the three techniques according to the difficulty of the task. The results obtained match with the quantitative results, most of the users (9) preferred 2D Cursor followed by the 
Torch (3). We also asked them whether the motion parallax effect made more difficult to them to select targets. Nine users reported no additional difficulties when the motion parallax was enabled while three reported difficulties. Notice that the ANOVA did not found significant differences in terms of performance nor accuracy for the camera factor.

For the second task (ordering), users were also asked to sort the techniques according to how they helped to perform the sorting task. As expected, the $2 D$ Cursor got the lowest score for all users, nine users preferred the Hand Avatar and three the Torch. Regarding pseudo-motion parallax effect, all users find it helpful to sort the objects.

\subsection{Discussion}

\subsubsection{Selection Task}

The results showed a main effect of the technique employed regarding pointing performance. In average, the performance raised from 0.8 seconds per object for the $2 D$ Cursor to 0.9 and 1.1 seconds per object for the Torch and the Hand Avatar respectively. Which are the main factors that decreased performance? According to the optimized initial impulse model [19], acquisition tasks are subdivided in a two-step movement phases. First a fast and inaccurate movement is made towards the target (ballistic phase) and then, iterative slow correction movements are executed until the target is acquired (corrective phase). For ballistic movements, the visual feedback provided by all three conditions provided enough information as the user does not need to be accurate. However, during the corrective phase the differences on visual feedback arose. Users required additional corrective movements when employing a 3D cursor. A second explanation, is that reaction time increased. We noticed that far smaller targets, some users required additional time to confirm that the 3D cursor was over the desired target, while for the 2D Cursor the confirmation time was less. This can be partially explained by the level of expertise of the users interacting with a $2 D$ Cursor.

Regarding pointing accuracy, the Hand Avatar condition presented the highest pixel deviation $(20.3 \mathrm{px})$ followed by the $2 D$ Cursor (14.8 px) and the Torch (13.6 px) (all differences were significant). First, notice that 20 pixels in a 24 inch monitor with a $1920 \times 1200$ represents approximately $5.5 \mathrm{~mm}$ and users were not instructed to aim to the center of objects. According to the visual feedback of the techniques (see Figure 9), the results of the Hand Avatar were expected, as it does not provide precise visual information about the selection point. However, it does provide enough information to know which is the object being pointed to. Interestingly, there were also significant differences between the $2 \mathrm{D} \mathrm{Cur-}$ sor and the Torch, being the Torch significantly more accurate. One possible explanation is that the alignment between the visual feedback and the spheres was easier with the specular higlight of the Torch. Also, the trade-of between speed and accuracy might be responsible, as users were slower when selecting with the Torch.

When analyzing the results obtained for the camera condition, we were quite surprised. It showed that the impact of motion parallax both in terms of performance and accuracy was non-significant, thus rejecting our hypotheses $\mathrm{H} 1.2$ and H1.4. While the camera movement might be a hindrance for the pointing, the users were able to compensate for the camera movement during the aiming process. Also, changes introduced by the pseudo-motion parallax effect on the index of difficulty of the task, decrease of $D$ and $W$, did not affect user performance. The decrease of the size of the target in control space was compensated by the decrease of the distance towards the target.

\subsubsection{Ordering Task}

During the ordering task, our main concern was to explore whether users were able to gather additional depth information from the environment. As we asked them to obtain the best ordering possible,
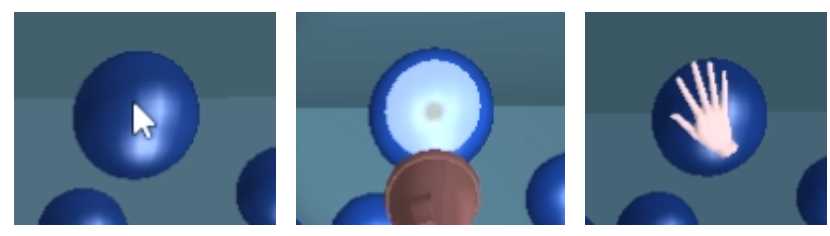

Figure 9: Although the underlying selection tool is a mouse pointer for all three techniques, the level of accuracy is determined by the visual feedback of the 2D Cursor (left), the Torch (center) and the Hand Avatar (right).

the ordering task forced the users to retrieve as much as depth information as possible. The results showed that in comparison with the base line condition ( $2 D$ Cursor + static camera), users were able to retrieve additional depth information with the 3D Cursors and the pseudo-motion parallax effect. Although the camera condition resulted in significant differences in ordering error, this could be biased by the poor performance for the combination of $2 \mathrm{D}$ and $\mathrm{SC}$ (see Figure 8 bottom). However, if we remove the $2 D$ Cursor from the ANOVA study, the camera factor still presents a main effect $(p<0.01 ; F=7.80)$.

On the one hand, 3D cursors, increased the depth perception of users, they allowed the users to explore the virtual environment in order to determine which objects were closer. Users had to "touch all the spheres and infer they relative depth according to the size of the $3 \mathrm{D}$ cursor; the 3D cursor projection gets smaller as the depth increases (see Figure 2). The depth sorting was also influenced by the depth disparity among objects. Objects that have similar depth position were harder to sort than those with great depth disparity. Regarding both techniques, the quantitative and qualitative results indicate that the Hand Avatar provided more precise depth cues than the Torch. As a counterpart, users had to examine all the objects multiple times in order to determine which objects were closer.

On the other hand, users were able to exploit the motion parallax effect to sort objects. Even when using the 2D cursor, users were able to achieve comparable results as with the 3D Cursors. During the motion parallax condition, in combination with the Hand Avatar and the $2 D$ Cursor, users significantly employed less time to gather depth information. The motion parallax allowed users to get an impression of the overall layout of the scene with just a few wide mouse movements. That information allowed users to distinguish objects with a great depth disparity. The need for "touching" all the objects was reduced (specially to sort objects with greater depth disparity).

\section{CONCLUSIONS AND FUtURE WORK}

The selection and manipulation of objects in a DVEs is typically achieved through a 2D cursor controlled with the mouse as an input device. In this paper, we have proposed and evaluated new techniques for improving users' depth perception when interacting with 3D content in desktop virtual environments. Proposed techniques provide a more ecological way to interact with $3 \mathrm{D}$ content using a mouse pointer as the visual feedback is adapted to the 3D content.

The Torch combines the spot light and the specular highlight which provide additional depth cues, with a 2D Cursor (the target point) to increase accuracy and an avatar of a lantern to reinforce the metaphor. The Hand Avatar displays a hand avatar placed and rotated according to the surface of the intersected 3D object, creating a pseudo-illusion of "touch". The size of the projected hand avatar also provide additional depth cues. Finally, the pseudo-motion parallax effect provides a translation of the virtual camera according to the mouse displacement. In contrast to the 3D cursors, the camera motion provides depth information for all the objects in the 3D scene at the same time. The pseudo-motion parallax can be easily 
combined with any $2 \mathrm{D}$ pointing selection technique.

The user evaluation showed that both 3D cursors significantly increased the users' depth perception, but at the expense of increase the selection time and decrease the accuracy. In contrast, the pseudo-motion parallax effect allowed users to gather additional depth information without decreasing users' performance. From these results we can state, that if we want to employ these techniques in a selection intensive application, it is preferred to enhance depth perception by using the pseudo-motion parallax effect in combination with a standard $2 \mathrm{D}$ mouse cursor. In contrast, in scenarios where the selection performance is not a requirement, the proposed 3D cursors are preferred as they provide improved depth perception and more information about the shape and size of the object. In addition, most of the users preferred the visual feedback of the Hand avatar and the Torch over the 2D cursor.

An additional advantage of the proposed techniques, is that they are compliant with 3D stereoscopic content. While 2D cursors are discouraged for stereoscopic content [3], mainly due to the limited ability of the human visual system to fuse objects with different retinal disparities, the proposed 3D cursors and the pseudo-motion parallax effect do not present any apparent limitation. 3D cursors are common in VR applications and motion parallax is a strong depth cue in combination with binocular vision.

Several directions can be pursued to extend our work. Selection performance of 3D cursors can be enhanced in order to obtain comparable performance ratios as standard 2D cursors. The visual feedback has to be enhanced in order to provide fast and accurate corrective movements. Second, the evaluation of the motion parallax only considered one mapping between mouse displacement and virtual camera movement. It would be interesting to explore which is the best mapping to trade-off camera movement and depth perception, and evaluate environments with different depth ranges. Finally, it would be also interesting to measure how realistic shadows in the scene can affect the depth perception in our proposed metaphors.

\section{ACKNOWLEDGEMENTS}

This work is supported by the W3D project, funded by Brittany region and Images et Reseaux French cluster.

\section{REFERENCES}

[1] Unity Technologies - Unity 3D - http://unity3d.com/.

[2] R. A. Andersen and D. C. Bradley. Perception of three-dimensional structure from motion. Trends in Cognitive Sciences, 2(6):222-228, 1998.

[3] F. Argelaguet and C. Andujar. Visual feedback techniques for virtual pointing on stereoscopic displays. In Proceedings of the 16th ACM Symposium on Virtual Reality Software and Technology, VRST '09, pages 163-170. ACM, 2009.

[4] R. Balakrishnan. "Beating" Fitts' Law: Virtual enhancements for pointing facilitation. International Journal of Human-Computer Studies, pages 857-874, 2004.

[5] D. A. Bowman, E. Kruijff, J. J. LaViola, and I. Poupyrev. 3D User Interfaces: Theory and Practice. Addison Wesley, 2004.

[6] F. Brard, J. Ip, M. Benovoy, D. El-Shimy, J. R. Blum, and J. R. Cooperstock. Did Minority Report get it wrong? Superiority of the mouse over 3D input devices in a 3D placement task. In IFIP Conference on Human-Computer Interaction, INTERACT, page 400, 2009.

[7] G. de Haan, M. Koutek, and F. H. Post. IntenSelect: Using dynamic object rating for assisting 3D object selection. Eurographics Symposium on Virtual Environments, pages 201-209, 2005.

[8] N. Elmqvist and J.-D. Fekete. Semantic pointing for object picking in complex 3d environments. In Proceedings of graphics interface 2008 , GI '08, pages 243-250, 2008.

[9] P. Fitts. The information capacity of the human motor system is controlled by the amplitude of movement. Journal of Experimental Psychology, 6(47):381-391, 1954.
[10] S. Frees, G. D. Kessler, and E. Kay. PRISM interaction for enhancing control in immersive virtual environments. ACM Transactions on Computer-Human Interaction, 14(1):2, 2007.

[11] T. Grossman and R. Balakrishnan. The design and evaluation of selection techniques for 3D volumetric displays. In UIST '06: Proceedings of the 19th annual ACM symposium on User interface software and technology, pages 3-12. ACM, 2006.

[12] K. P. Herndon, A. van Dam, and M. Gleicher. The challenges of 3D interaction: a CHI '94 workshop. SIGCHI Bull., 26(4):36-43, 1994.

[13] H. Hu, A. Gooch, S. Creem-Regehr, and W. Thompson. Visual cues for perceiving distances from objects to surfaces. Presence: Teleoperators and Virtual Environments, 11(6):652-664, 2002.

[14] G. S. Hubona, P. N. Wheeler, G. W. Shirah, and M. Brandt. The relative contributions of stereo, lighting, and background scenes in promoting $3 \mathrm{~d}$ depth visualization. ACM Trans. Comput.-Hum. Interact., 6:214-242, September 1999.

[15] C. R. J.D. Boeck, T. D. Weyer and K. Coninx. Using the non-dominant hand for selection in 3D. In IEEE Symposium on 3D User Interfaces (3DUI), pages 53-58, 2006.

[16] A. Kadri, A. Lecuyer, and J.-M. Burkhardt. The visual appearance of user's avatar can influence the manipulation of both real devices and virtual objects. In IEEE Symposium on $3 D$ User Interfaces (3DUI), pages $291-298,2007$.

[17] M. Kitazaki, H. Kobiki, and L. T. Maloney. Effect of pictorial depth cues, binocular disparity cues and motion parallax depth cues on lightness perception in three-dimensional virtual scenes. PLoS ONE, 3(9):e3177, 092008.

[18] R. Kopper, F. Bacim, and D. Bowman. Rapid and accurate $3 \mathrm{~d}$ selection by progressive refinement. In IEEE Symposium on $3 D$ User Interfaces (3DUI), pages 67-74, 2011.

[19] D. E. Meyer, R. A. Abrams, S. Kornblum, C. E. Wright, and J. E. K. Smith. Optimality in human motor performance: ideal control of rapid aimed movements. Psychological Review, 95:340-370, 1988.

[20] J. G. Phillips, J. W. Meehan, and T. J. Triggs. Effects of cursor orientation and required precision on positioning movements on computer screens. International Journal of Human-Computer Interaction, 15:379-389, 2003.

[21] B. A. Po, B. D. Fisher, and K. S. Booth. Comparing cursor orientations for mouse, pointer, and pen interaction. In Proceedings of the SIGCHI conference on Human factors in computing systems, CHI '05, pages 291-300. ACM, 2005.

[22] I. Poupyrev, S. Weghorst, M. Billinghutst, and T. Ichikawa. Egocentric object manipulation in VEs: Empirical evaluation of interaction techniques. Computer Graphics Forum, 3(17):41-52, 1998.

[23] B. Rogers and M. Graham. Motion parallax as an independent cue for depth perception. Perception, 8(2):125-134, 1979.

[24] K. Sons, F. Klein, D. Rubinstein, S. Byelozyorov, and P. Slusallek. XML3D : interactive 3D graphics for the web. In 15th international Conference on Web 3D Technology, page 175, 2010.

[25] F. Stenicke, T. Ropinski, G. Bruder, and K. Hinrichs. Interscopic user interface concepts for fish tank virtual reality systems. Virtual Reality Conference, IEEE, pages 27-34, 2007.

[26] L. Vanacken, T. Grossman, and K. Coninx. Exploring the effects of environment density and target visibility on object selection in $3 \mathrm{D}$ virtual environments. pages 115-122, 2007.

[27] L. Vanacken, C. Raymaekers, and K. Coninx. Evaluating the influence of multimodal feedback on egocentric selection metaphors in VEs. Haptic and Audio Interaction Design, pages 12-23, 2006.

[28] L. Wanger. The effect of shadow quality on the perception of spatial relationships in computer generated imagery. In Proceedings of the Symposium on Interactive 3D graphics, pages 39-42, 1992.

[29] L. Wanger, J. Ferwerda, and D. Greenberg. Perceiving spatial relationships in computer-generated images. IEEE Computer Graphics and Applications, 12(3):44 -58, 1992.

[30] C. Ware and G. Franck. Evaluating stereo and motion cues for visualizing information nets in three dimensions. ACM Transactions on Graphics, 15:121-140, 1996. 